Also available at http://amc.imfm.si

ISSN 1855-3966 (printed edn.), ISSN 1855-3974 (electronic edn.)

ARS MATHEMATICA CONTEMPORANEA 4 (2011) 271-289

\title{
The symmetric genus spectrum of finite groups
}

\author{
Marston D. E. Conder* \\ Mathematics Department, University of Auckland \\ Private Bag 92019, Auckland 1142, New Zealand \\ Thomas W. Tucker \\ Mathematics Department, Colgate University, Hamilton, NY 13346, USA
}

Received 10 December 2009, accepted 16 July 2011, published online 9 August 2011

\begin{abstract}
The symmetric genus of the finite group $G$, denoted by $\sigma(G)$, is the smallest nonnegative integer $g$ such that the group $G$ acts faithfully on a closed orientable surface of genus $g$ (not necessarily preserving orientation). This paper investigates the question of whether for every non-negative integer $g$, there exists some $G$ with symmetric genus $g$. It is shown that that the spectrum (range of values) of $\sigma$ includes every non-negative integer $g \not \equiv 8$ or $14 \bmod 18$, and moreover, if a gap occurs at some $g \equiv 8$ or 14 modulo 18 , then the prime-power factorization of $g-1$ includes some factor $p^{e} \equiv 5 \bmod 6$. In fact, evidence suggests that this spectrum has no gaps at all.
\end{abstract}

Keywords: Symmetric genus, Riemann surface, Riemann-Hurwitz equation, NEC group, signature.

Math. Subj. Class.: 57M60; 14H37, 20B25, 20F38, $20 H 10$

\section{Introduction}

The automorphisms of a compact Riemann surface $S$ of genus $g>1$ depend on $g$ (and on the analytic structure of $S$ ). A celebrated theorem of Hurwitz (1893) states that if $G$ is any group of automorphisms of such a surface preserving orientation, then $|G| \leq 84(g-1)$; see $[20,18]$. Similarly, Wiman's theorem $[19,35]$ shows that $|G| \leq 2(2 g+1)$ when $G$ is cyclic. Both of these bounds are easy consequences of what is now known as the Riemann-Hurwitz equation. They also stimulated work by Burnside and Maschke and others on the actions of specific kinds of groups on surfaces, and on embeddings of graphical representations of groups on surfaces; see [5, 26].

\footnotetext{
* Supported in part by the N.Z. Marsden Fund (grant no. UOA 721).

E-mail addresses: m.conder@auckland.ac.nz (Marston D. E. Conder), ttucker@colgate.edu (Thomas W. Tucker)
} 
Shortly afterwards, Brahana [4] initiated the study of regular maps on surfaces, which generalize the Platonic solids. A map is a dissection of a surface into vertices, edges and faces, and is called regular (or more technically, rotary) if it has symmetries that act as full cyclic rotations about any face and any vertex.

These things are now much better understood than in the early 1900s. It is now known exactly which kinds of groups arise as finite groups of homeomorphisms of the sphere (genus 0) and the torus (genus 1); see [18]. Also much is known about the largest finite groups of automorphisms of a surface of given genus; see [1, 25, 9, 12] for example. Significant progress has been made recently through the study of Fuchsian groups and nonEuclidean crystallographic groups (see [23]) and their quotients, with the help of advanced methods such as voltage graph constructions (see [18]) and computation (see [10] for example). Further relevant comments will be made in the final section.

Given a finite group $G$, one may ask what is the smallest non-negative integer $g$ for which the group $G$ acts faithfully on a closed orientable surface of genus $g$. The smallest such $g$ is now known as the symmetric genus of $G$, and denoted by $\sigma(G)$; see [18, 32]. The definition allows $G$ to contain both orientation-preserving and orientation-reversing elements, and if we require that the action be orientation-preserving, then we have the strong symmetric genus, denoted by $\sigma^{o}(G)$. Similarly, for closed non-orientable surfaces we have the symmetric cross-cap number, denoted by $\tilde{\sigma}(G)$.

Determination of $\sigma^{o}(G)$ and/or $\sigma(G)$ for various candidates for $G$ was a question of interest to Burnside et al, and has been answered for several classes of finite groups, including cyclic and dihedral groups (of strong symmetric genus 0), abelian groups [24, 27], the alternating and symmetric groups [7, 8], other finite Coxeter groups [21], groups of odd order [29], the projective special linear groups $L_{2}(q)$ [17], and the sporadic finite simple groups (see [14, 34] and other references therein).

A further natural question to ask is this:

Question 1.1. For every integer $g \geq 0$, is there is a finite group $G$ with $\sigma(G)=g$ ? In other words, for all $g \geq 0$, is there some group $G$ that acts faithfully on a closed orientable surface of genus $g$, but on no such surface of genus smaller than $g$ ?

The analogous question for the strong symmetric genus $\sigma^{o}(G)$ has been answered in the affirmative by May and Zimmerman [28], who showed that $\sigma^{\circ}(G)$ takes all possible integer values $g \geq 0$ when $G$ is restricted to the family of direct products $C_{m} \times D_{n}$ (of order 2mn). Subsequently, Etayo and Martinez [16] have shown that the same family gives values of the symmetric cross-cap number $\tilde{\sigma}$ that cover a significant proportion of the positive integers.

On the other hand, $\sigma\left(C_{m} \times D_{n}\right)=1$ for all $m$ and $n$, since these groups act faithfully on the torus (with action of type (h) in the classification of toroidal groups given in [18]), so these groups are of no use for answering Question 1.1 for $\sigma$.

We can, however, show that the spectrum (range of values) of the symmetric genus contains well over $88 \%$ of all positive integers. Specifically, in this paper we prove the following:

Theorem 1.2. If $g$ is any non-negative integer such that $g \not \equiv 8$ or $14 \bmod 18$, then there exists a finite group $G$ with symmetric genus $\sigma(G)=g$. Moreover, the same holds if $g \equiv 8$ or 14 modulo 18 and every factor $p^{e}$ in the prime-power factorization of $g-1$ is congruent to $1 \bmod 6$. 
Hence the only possible gaps in the spectrum for $\sigma$ (if there are any gaps at all) must occur at integers $g \equiv 8$ or $14 \bmod 18$ such that $g-1$ has a factor $p^{e} \equiv 5 \bmod 6$ in its primepower factorization. Evidence suggests, however, that there are no gaps. In fact we have found none, and so we join May and Zimmerman [28] in making the following conjecture:

Conjecture 1.3. For every integer $g \geq 0$, there is a finite group $G$ with $\sigma(G)=g$.

The challenge in proving this conjecture is the difficulty of finding families of groups whose orders grow in a well-behaved way and whose symmetric genera can be computed. Knowing the symmetric genus of particular families of groups is helpful, but often only to a certain extent. For the alternating and symmetric groups for example, it is known that $\sigma^{o}\left(A_{n}\right)=\sigma\left(A_{n}\right)=\sigma\left(S_{n}\right)=n ! / 168+1$ for all but finitely many $n$ (as follows from [6]), but the orders of these groups (and apparently other Hurwitz groups [9]) grow exponentially. We have found several good families of groups whose orders grow in arithmetic progression, as we will demonstrate in the proof of Theorem 1.2, but sadly, there are not quite enough of them.

The organization of this paper is as follows. In Section 2, we provide some further background on the Riemann-Hurwitz equation, signatures of group actions, and non-Euclidean crystallographic groups. In Section 3 we show that $\sigma(G)$ can be any odd positive integer. Then in Section 4 we consider all even genera $g \not \equiv 8,14 \bmod 18$, while in Section 5 we handle those $g \equiv 8,14 \bmod 18$ for which $g-1$ has no factor $p^{e} \equiv 5 \bmod 6$ in its primepower factorization. Finally, in Section 6, we consider the remaining gaps in the spectrum of $\sigma$, and other matters such as the genus spectra of various kinds of regular maps, and the spectrum of the plain (Cayley) genus of a group, as defined by White [33].

\section{Signatures and the Riemann-Hurwitz equation}

Let $G$ be a finite group of homeomorphisms of a closed orientable surface $S$. We consider three possibilities: one where the action of $G$ has a finite number of fixed points on $S$, which happens when all elements of $G$ preserve orientation; a second where the action includes reflections (which are involutory orientation-reversing homeomorphisms that fix at least one simple close curve in $S$ ) and the quotient surface $S / G$ (obtained by identifying each orbit of $S$ under the action of $G$ to a point) is orientable; and a third where the action includes orientation-reversing homeomorphisms but the quotient surface $S / G$ is non-orientable.

In the first case, the Riemann-Hurwitz equation says

$$
\chi(S)=|G|\left(\chi(S / G)-\sum_{i=1}^{r}\left(1-1 / m_{i}\right)\right),
$$

where $\chi$ denotes the Euler characteristic of the relevant surface, and $m_{1}, \ldots, m_{r}$ are the orders of the branch points in $S / G$. (Here the natural map $S \rightarrow S / G$ is a branched covering.) This equation is a simple consequence of counting vertices, faces, and edges of an appropriate dissection of the surface $S / G$, taking into account that the $i$ th branch point has only $|G| / m_{i}$ preimages in $S$ and hence has a deficiency of $|G|\left(1-1 / m_{i}\right)$, for $\left.1 \leq i \leq r\right)$. In terms of the genus, the equation says

$$
g=1+\frac{|G|}{2}\left(2 \gamma-2+\sum_{i=1}^{r}\left(1-1 / m_{i}\right)\right),
$$


where $g$ is the genus of $S$ and $\gamma$ is the genus of $S / G$ (given by $\chi(S)=2-2 g$ and $\chi(S / G)=2-2 \gamma)$.

In this case, we say that the group $G$ acts with signature $\left(\gamma ;+;\left[m_{1}, \ldots, m_{r}\right] ;\{-\}\right)$. Associated with this signature is a finitely-presented group $\Gamma$, called a Fuchsian group, of the form

$$
\Gamma=\left\langle a_{1}, b_{1}, \ldots, a_{\gamma}, b_{\gamma}, x_{1}, \ldots, x_{r} \mid x_{1}^{m_{1}}=\ldots=x_{r}^{m_{r}}=\left[a_{1}, b_{1}\right] \ldots\left[a_{\gamma}, b_{\gamma}\right] x_{1} \ldots x_{r}=1\right\rangle,
$$

with the property that $G$ is a quotient of $\Gamma$, under an epimorphism $\theta: \Gamma \rightarrow G$ that preserves the orders $m_{i}$ of the generators $x_{i}$. Such an epimorphism is often called smooth. From the uniformization viewpoint, $G \cong \Gamma / \Lambda$ and $S$ is the quotient space $\mathcal{U} / \Lambda$ of the upper half-plane $\mathcal{U}$ by $\Lambda=\operatorname{ker} \theta$, which is the fundamental group of $S$.

In the second case, which has reflections, the Riemann-Hurwitz equation says

$$
\chi(S)=|G|\left(\chi(S / G)-\sum_{i=1}^{r}\left(1-1 / m_{i}\right)-\sum_{j=1}^{k} \sum_{\ell=1}^{s_{j}}\left(1-1 / n_{j \ell}\right) / 2\right),
$$

where the quotient surface $S / G$ has $k(>0)$ boundary components, the $m_{i}$ are the orders of the $r$ branch points in the interior of $S / G$, and the $n_{j \ell}$ are the orders of the $s_{j}$ branch points on the $j$ th boundary component $C_{j}$ of $S / G$, for $1 \leq j \leq k$. This is again a simple consequence of counting vertices, faces, and edges, but here a branch point of order $n_{j \ell}$ on the boundary has deficiency $|G|\left(1-1 / n_{j \ell}\right) / 2$. As before, the Riemann-Hurwitz equation can also be re-expressed in terms of the genera of $S$ and $S / G$, which are $\chi(S)=2-2 g$ and $\chi(S / G)=2-2 \gamma-k$.

In this case, the signature is $\left(\gamma ;+;\left[m_{1}, \ldots, m_{r}\right] ;\left\{\left(n_{11}, \ldots, n_{1 s_{1}}\right), \ldots,\left(n_{k 1}, \ldots, n_{k s_{k}}\right)\right\}\right)$, and associated with it is a non-Euclidean crystallographic group (or NEC group) $\Gamma$, generated by elements $a_{1}, b_{1}, \ldots, a_{\gamma}, b_{\gamma}, x_{1}, \ldots, x_{r}, e_{1}, \ldots, e_{k}, c_{10}, \ldots, c_{1 s_{1}}, \ldots, c_{k 0}, \ldots, c_{k s_{k}}$ subject to defining relations

$$
\begin{gathered}
x_{i}^{m_{i}}=1 \text { for } 1 \leq i \leq r, \quad c_{j \ell}^{2}=1 \text { for } 1 \leq j \leq k \text { and } 0 \leq \ell \leq s_{j}, \\
e_{j} c_{j 0} e_{j}^{-1}=c_{j s_{j}} \text { for } 1 \leq j \leq k, \quad\left(c_{j(\ell-1)} c_{j \ell}\right)^{n_{j \ell}}=1 \text { for } 1 \leq j \leq k \text { and } 1 \leq \ell \leq s_{j}, \\
\text { and }\left[a_{1}, b_{1}\right] \ldots\left[a_{\gamma}, b_{\gamma}\right] x_{1} \ldots x_{r} e_{1} \ldots e_{k}=1 .
\end{gathered}
$$

Again $G$ is a (smooth) quotient of $\Gamma$, under an epimorphism $\theta: \Gamma \rightarrow G$ that preserves the orders of the generators and their products when these orders are specified, and $S$ is the quotient space $\mathcal{U} / \Lambda$ where $\Lambda=\operatorname{ker} \theta$ is the fundamental group of $S$. This time, however, there is another requirement: $\Gamma$ has a unique subgroup $\Gamma^{o}$ of index 2 that contains all of $a_{1}, b_{1}, \ldots, a_{\gamma}, b_{\gamma}, x_{1}, \ldots, x_{r}, e_{1}, \ldots, e_{k}$ but none of the involutory generators $c_{j \ell}$, and this subgroup is taken by the epimorphism $\theta$ to the orientation-preserving subgroup of $G$, necessarily of index 2 in $G$.

The third case is similar to the second, but with the ' + ' replaced by '-' in the signature, the generators $a_{1}, b_{1}, \ldots, a_{\gamma}, b_{\gamma}$ of $\Gamma$ replaced by generators $d_{1}, \ldots, d_{\gamma}$, and the final defining relation for $\Gamma$ replaced by

$$
d_{1}^{2} \ldots d_{\gamma}^{2} x_{1} \ldots x_{r} e_{1} \ldots e_{k}=1,
$$

while $\Gamma^{o}$ is taken as the unique subgroup of index 2 containing all of $x_{1}, \ldots, x_{r}, e_{1}, \ldots, e_{k}$ but none of $d_{1}, . ., d_{\gamma}$ and none of the involutory generators $c_{j \ell}$. The Riemann-Hurwitz equation is the same as in the second case, except that $\chi(S / G)=2-\gamma-k$. 
These observations work in reverse, in the sense that if $G$ is any smooth quotient of a non-Euclidean crystallographic group $\Gamma$, then $G$ acts faithfully on a closed surface whose characteristic is given by the Riemann-Hurwitz equation.

Now in each of the theorems in the next few sections of this paper, we will exhibit a faithful action of a particular finite group $G$ on an orientable surface $S^{\prime}$ of genus $g^{\prime}$, and then show $G$ has no faithful action on any surface $S$ of genus $g$ smaller than $g^{\prime}$.

In all cases, except where otherwise noted, we will have $|G| \geq 4\left(g^{\prime}-1\right)$, and so to improve on the genus $g^{\prime}$, we would require $g<g^{\prime} \leq 1+|G| / 4$. Linking this bound with the Riemann-Hurwitz equation puts a strong restriction on the parameters involved in the signatures of possible group actions, and hence also on the NEC-groups that can give rise to such actions of $G$ within the desired genus range.

Such restrictions are illustrated in the following proposition, which will be useful for us later. Note that in each case the genus $g$ is given by $g=1+|G| \xi / 2$, for some rational number $\xi$ that depends on the signature of the corresponding action. If $\xi$ is negative then $g=0$ and the group $G$ acts on the sphere, while if $\xi=0$ then $g=1$ and the action is toroidal.

Proposition 2.1. Let $G$ be a finite group that is neither cyclic nor abelian of rank 2, nor generated by involutions, and suppose $G$ acts faithfully on an orientable surface of genus $g=1+|G| \xi / 2$ where $\xi<1 / 2$. Then one of the cases below occurs:

(A) $G$ acts with signature $\left(0 ;+;\left[m_{1}, \ldots, m_{r}\right] ;\{-\}\right)$, where $r=3$ or 4 , and $\xi=r-2-\sum_{i=1}^{r} 1 / m_{i}$

(B1) G acts with signature $\left(0 ;+;[m] ;\left\{\left(q_{1}, \ldots, q_{s}\right)\right\}\right)$ where $s=1$, 2 or 3 , and $\xi=s / 2-1 / m-\sum_{j=1}^{s} 1 /\left(2 q_{j}\right)$;

(B2) $G$ acts with signature $\left(0 ;+;\left[m_{1}, m_{2}\right] ;\{(q)\}\right)$ where $m_{1}=2$ or $q=1$, and $\xi=3 / 2-1 / m_{1}-1 / m_{2}-1 /(2 q)$

(B3) $G$ acts with signature $(0 ;+;[-] ;\{(1),(q)\})$, and $\xi=1 / 2-1 /(2 q)$;

(C1) $G$ acts with signature $\left(1 ;-;\left[m_{1}, m_{2}\right] ;\{-\}\right)$, and $\xi=1-1 / m_{1}-1 / m_{2}$;

(C2) Gacts with signature $(1 ;-;[-] ;\{(q)\})$, and $\xi=1 / 2-1 /(2 q)$;

(C3) $G$ acts with signature $(2 ;-;[-] ;\{-\})$, and $\xi=1$.

In cases (B1), (B3) and (C2), and in case (B2) with $m_{1}=2$, the quotient $G / J$ of $G$ by the normal subgroup $J$ generated by involutions is cyclic, while in case $(\mathrm{C} 3)$, and in cases (B3) and (C2) with $q=1$, the quotient $G / Z(G)$ is cyclic or dihedral, and the group $G$ has a subgroup of index 2 that is abelian of rank at most 2 .

Proof. First suppose the action of $G$ preserves orientation. Then the signature is $(\gamma ;+$; $\left.\left[m_{1}, \ldots, m_{r}\right] ;\{-\}\right)$, and we need $\left.2 \gamma-2+\sum_{i=1}^{r}\left(1-1 / m_{i}\right)\right)<1 / 2$, which forces $\gamma=0$ or 1 (so $S / G$ is the sphere or the torus). But also $1-1 / m_{i} \geq 1 / 2$ for all $i$, and hence we find that if $\gamma=0$ then $r \leq 4$, while if $\gamma=1$ then $r=0$. If $\gamma=0$ and $r \leq 1$ then the NEC-group $\Gamma$ is trivial, while if $\gamma=0$ and $r=2$ then $\Gamma$ is cyclic (generated by elements $x_{1}$ and $x_{2}$ such that $x_{1} x_{2}=1$ ), and this case can be ruled out since $G$ is not cyclic. Similarly, if $\gamma=1$ and $r=0$ then $\Gamma$ is abelian of rank at most 2 (generated by elements $a_{1}$ and $b_{1}$ such that $\left[a_{1}, b_{1}\right]=1$ ), so this case is ruled out too. Thus $S / G$ is the sphere, with three or four branch points, giving case (A). 
Next, suppose the action has reflections and $S / G$ is orientable. Then the RiemannHurwitz equation gives $\chi(S / G)=2 \gamma+k-2<1 / 2$ where $k \geq 1$, so $\gamma=0$ and $k=1$ or 2 ; in other words, $S / G$ is the sphere, and there can be only one or two boundary components. Moreover, if $k=1$ then if $s$ is the total number of branch points of order greater than 1 on the boundary component, then $2 r+s \leq 5\left(\right.$ since $\left(1-1 / n_{j \ell}\right) / 2 \geq 1 / 4$ whenever $n_{j \ell}>1$ ). When $r=0$ the NEC-group $\Gamma$ is generated by involutions (which induce reflections of $S$ ), and so we can rule out that case; when $r=1$ we have case (B1); and when $r=2$ we have (B2), where we note that if $m_{2} \geq m_{1} \geq 3$ and $q>1$ then $\xi=3 / 2-1 / m_{1}-1 / m_{2}-1 /(2 q) \geq 7 / 12>1 / 2$, which is impossible. Similarly, if $k=2$ then $r=0$ and $s \leq 1$, giving (B3).

In case (B1), the NEC group $\Gamma$ is generated by $s$ involutions $c_{0}, \ldots, c_{s-1}$ and an element $x$ such that $x^{m}=\left(c_{0} c_{1}\right)^{q_{1}}=\cdots=\left(c_{s-2} c_{s-1}\right)^{q_{s-1}}=\left(c_{s-1} x^{-1} c_{0} x\right)^{q_{s}}=1$. In particular, the quotient of $\Gamma$ by the subgroup generated by its involutions is cyclic (generated by the image of $x$ ), and so the analogous property holds in $G$. Similarly, in case (B2), the NEC group $\Gamma$ is generated by an involution $c$ and two elements $x_{1}$ and $x_{2}$ such that $x_{1}{ }^{m_{1}}=x_{2}{ }^{m_{2}}=\left[c, x_{1} x_{2}\right]^{q}=1$, and so if $q=1$ then the quotient of $G$ by the subgroup generated by its involutions is cyclic. In case (B3), the NEC group $\Gamma$ is generated by two involutions $c_{1}$ and $c_{2}$ and an element $e$ such that $\left[e, c_{1}\right]=\left[e, c_{2}\right]^{q}=1$, so again the quotient of $G$ by the subgroup generated by its involutions is cyclic. Moreover, if $q=1$ then $e$ is central and so $G / Z(G)$ is cyclic or dihedral (generated by the images of $c_{1}$ and $c_{2}$ ), and also the orientation-preserving subgroup of $G$ is the image of the index 2 subgroup of $\Gamma$ generated by $e$ and $c_{1} c_{2}$, which is abelian.

Finally, suppose $S / G$ is non-orientable. Then the Riemann-Hurwitz equation gives $\chi(S / G)=\gamma+k-2<1 / 2$, where $\gamma \geq 1$. If $\gamma=1$ and $k=0$ then $r \leq 2$, but if $r=0$ or 1 then the NEC group $\Gamma$ is cyclic, so we have $r=2$, which gives case (C1). If $\gamma=k=1$ then $r=0$, and moreover, there can be at most one branch point of order greater than 1 on the boundary component, and so we have (C2). The only other possibility is $\gamma=2$ and $k=0$, and then $r=0$ and we have (C3).

In case (C2), the NEC group $\Gamma$ (and analogously, its quotient $G$ ) is generated by an involution $c$ and an element $d$ such that $\left[c, d^{2}\right]^{q}=1$, and again, it follows that the quotient of $G$ by the subgroup generated by its involutions is cyclic. Moreover, if $q=1$, then $d^{2}$ is central, so $G / Z(G)$ is cyclic or dihedral, and the index 2 subgroup $\left\langle d^{2}, c d\right\rangle$ (preserving orientation) is abelian. In case (C3), the NEC group $\Gamma$ is generated by two elements $d_{1}$ and $d_{2}$ such that $d_{1}^{2} d_{2}^{2}=1$. In particular, the element $d_{1}^{2}=d_{2}{ }^{-2}$ is central, making $G / Z(G)$ cyclic or dihedral, and the images of $d_{1}^{2}$ and $d_{1} d_{2}$ generate an abelian subgroup of index 2 in $G$ (preserving orientation).

\section{Odd genera}

In this section, we consider two families of groups defined as follows:

$$
\begin{aligned}
& U_{n}=\left\langle x, y \mid x^{4}=y^{4}=1,\left[x^{2}, y\right]=\left[y^{2}, x\right]=1,(x y)^{2 n}=1\right\rangle, \text { and } \\
& V_{n}=\left\langle x, y \mid x^{4}=y^{4}=1,\left[x^{2}, y\right]=\left[y^{2}, x\right]=1,(x y)^{2 n}=x^{2}\right\rangle,
\end{aligned}
$$

for each positive integer $n$.

Note that in each case, the subgroup $N$ generated by $x^{2}$ and $y^{2}$ is central, with dihedral quotient $\left\langle x, y \mid x^{2}=y^{2}=(x y)^{2 n}=1\right\rangle \cong D_{2 n}$, of order $4 n$, in which the image of $x y$ has order $2 n$. Similarly, the subgroup generated by $(x y)^{2}$ is normal, because the centrality of $x^{2}$ and $y^{2}$ implies that $(x y)^{2}=\left(x^{-1} y^{-1}\right)^{2}$ and hence that $(x y)^{2}$ is inverted by conjugation 
by each of $x$ and $y$. The quotient $U_{n} /\left\langle(x y)^{2}\right\rangle$ is the group $U_{1}$, which has a transitive permutation representation of degree 8 given by $x \mapsto(1,2,3,4)(5,6,7,8)$ and $y \mapsto$ $(1,6,5,2)(3,8,7,4)$, and this shows that in $U_{n}$, the subgroup generated by $x^{2}$ and $y^{2}$ has order 4 (and that $U_{1}$ has order 16). Thus $U_{n}$ has order $16 n$. Moreover, $V_{n}$ is isomorphic to the quotient of $U_{2 n}$ by its central subgroup of order 2 generated by $(x y)^{2 n} x^{-2}$, and therefore $V_{n}$ has order $16 n$ as well.

Next we make an important observation about the involutions in each of these groups. Again since $x^{2}$ and $y^{2}$ are central, every element $w$ in $G$ can be expressed in the form $(x y)^{i} z$ or $(x y)^{i} x z$ where $0 \leq i<2 n$ and $z \in\left\{1, x^{2}, y^{2}, x^{2} y^{2}\right\}$, and then

$$
w^{2}=\left((x y)^{i} z\right)^{2}=(x y)^{2 i} \quad \text { or } \quad\left((x y)^{i} x z\right)^{2}=x^{2 i+2} y^{2 i} .
$$

In the first case, $w$ is an involution only when $i=0$ or $n$, while in the second case, $w$ has order 4 since $w^{2}=x^{2}$ or $y^{2}$, depending on whether $i$ is even or odd (respectively). Hence the only involutions in $V_{n}$ are $x^{2}, y^{2}$ and $x^{2} y^{2}$, while the involutions in $U_{n}$ are the seven non-trivial elements of the form $z$ or $(x y)^{n / 2} z$ with $z \in\left\{1, x^{2}, y^{2}, x^{2} y^{2}\right\}$.

Theorem 3.1. $\sigma\left(V_{n}\right)=4 n-1$ for every integer $n>1$.

Proof. Let $G=V_{n}$ where $n>1$, and let $I$ be the set of elements of order 1 or 2 in $G$ (namely $I=\left\{1, x^{2}, y^{2}, x^{2} y^{2}\right\}$ ). The generating set $\left\{x, y,(x y)^{-1}\right\}$ gives an action of $G$ with signature $(0 ;+;[4,4,4 n] ;\{-\})$ on an orientable surface of genus

$$
1+8 n(1-1 / 4-1 / 4-1 /(4 n))=4 n-1,
$$

and we show that $G$ has no faithful action on a surface of genus $g$ smaller than this.

Any such action of $G$ on an orientable surface $S$ has no reflections, since all involutions in $G$ are squares or products of squares and so must preserve orientation. Hence only cases (A), (C1) or (C3) of Proposition 2.1 are applicable here.

We first consider case (A). If $r=3$, then $G$ has a generating set $\{u, v, w\}$ of three elements of orders $m_{1}, m_{2}, m_{3}$, with $u v w=1$. In the quotient $G /\left\langle x^{2}, y^{2}\right\rangle \cong D_{2 n}$, two of these three elements must have images of order 2 while the other has image of order $2 n$. Without loss of generality, $u$ and $v$ have images of order 2, so $u$ and $v$ have order 4 in $G$, and $w$ must have order $2 n$ in the quotient, so $w$ must be of the form $w=(x y)^{i} z$, where $i$ is relatively prime to $2 n$ and $z \in I$. In particular, $w$ has the same order as $x y$, namely $4 n$, and $\left[m_{1}, m_{2}, m_{3}\right]=[4,4,4 n]$, so the genus can only be $4 n-1$. Similarly, if $r=4$, then $G$ is generated by four elements $t, u, v, w$ of orders $m_{1}, m_{2}, m_{3}, m_{4}$ such that tuvw $=1$, but again, at least two of these generators must have order at least 2 in the dihedral quotient $G /\left\langle x^{2}, y^{2}\right\rangle$ and order at least 4 in $G$, which gives $g \geq 1+8 n(2-1 / 2-1 / 2-1 / 4-1 / 4)=$ $4 n+1>4 n-1$, a contradiction.

In case $(\mathrm{C} 1)$, the group $G$ is generated by two elements $d$ and $u$ such that $u^{m_{1}}=$ $\left(d^{2} u\right)^{m_{2}}=1$. The images of $u$ and $d$ in the dihedral quotient $G /\left\langle x^{2}, y^{2}\right\rangle \cong D_{2 n}$ have orders 2 and 2 , or 2 and $2 n$, in some order, and since $n \geq 2$ it follows that in $G$ these two elements both have order at least 4 . Thus $m_{1} \geq 4$. The same argument applies to $d$ and $u d^{2}$, so $m_{2} \geq 4$ as well. Thus $1 / m_{1}+1 / m_{2} \leq 1 / 2$, and so $g=1+8 n\left(1-1 / m_{1}-1 / m_{2}\right) \geq$ $1+8 n / 2>4 n-1$, a contradiction.

In case (C3), the group $G$ is generated by two elements $d_{1}$ and $d_{2}$ such that $d_{1}^{2} d_{2}^{2}=1$. Now $d_{1}$ and $d_{2}$ both have order greater than 2 (since $G$ is not cyclic or dihedral), but in the dihedral quotient $G /\left\langle x^{2}, y^{2}\right\rangle$, they must both have order 2 (since $d_{1}^{2}=d_{2}{ }^{2}$ ), and hence 
both have order 4 in $G$. It follows that the central subgroup $\left\langle d_{1}^{2}\right\rangle$ of $G$ has order 2 , with dihedral quotient of order $8 n$. The latter quotient, however, is also generated by the images of $x$ and $y$, and since $4 n>4$ the only way this can happen is for those images to both have order 2. But then $x^{2}$ and $y^{2}$ are both equal to $d_{1}^{2}$, which is impossible (since $\left\langle x^{2}, y^{2}\right\rangle$ has order 4).

Thus we can do no better than genus $4 n-1$, as claimed.

For the case $n=1$, we note that $V_{1}$ is generated by its elements $d_{1}=x$ and $d_{2}=x y$, with $d_{1}^{2} d_{2}^{2}=x^{2} x^{2}=1$. Since also the subgroup generated by $x^{2}$ and $y$ has index 2 but contains neither $x$ nor $x y$, it follows that $V_{1}$ acts with signature $(2 ;-;[-],\{-\})$ on an orientable surface of genus $g=1$ (that is, on the torus), and so $\sigma\left(V_{1}\right)=1$, rather than $4 n-1=3$. On the other hand, it is well known that the simple group $L_{2}(7)$ has symmetric genus 3 , and hence we have shown that for every positive integer $g \equiv 3 \bmod 4$, there exists a group of symmetric genus $g$.

The same is known to be true for all $g \equiv 1 \bmod 4$, since May and Zimmerman showed that the abelian group $\left(C_{2}\right)^{3} \times C_{2 m}$ of order $16 m$ has symmetric genus $4 m+1$, for every positive integer $m$; see [27]. We can add to these results the following:

Theorem 3.2. $\sigma\left(U_{n}\right)=4 n-3$ for every positive integer $n$.

Proof. The proof of this theorem is largely similar to that of the previous one. Let $G=U_{n}$, and let $I$ be the set of elements of order 1 or 2 in $G$, namely the eight elements of the form $z$ or $(x y)^{n} z$ with $z \in\left\{1, x^{2}, y^{2}, x^{2} y^{2}\right\}$. Note that the images of all these elements in the dihedral quotient $G /\left\langle x^{2}, y^{2}\right\rangle \cong D_{2 n}$ are central.

The generating set $\left\{x, y,(x y)^{-1}\right\}$ gives an action with signature $(0 ;+;[4,4,2 n] ;\{-\})$ on an orientable surface of genus

$$
1+8 n(1-1 / 4-1 / 4-1 /(2 n))=4 n-3 .
$$

When $n=1$ this action is toroidal, and as $U_{1}$ has no faithful action on the sphere (by Maschke's theorem), we may assume that $n>1$. We now suppose that $G$ has a faithful action on some surface $S$ of genus smaller than $4 n-3$.

If $G$ acts on $S$ without reflections, then the same kind of arguments as made in the proof of Theorem 3.1 lead only to contradictions, whether $S / G$ is orientable or non-orientable. If, instead, $G$ acts with reflections, then the images of all involutions in $G$ are central in the dihedral quotient $G /\left\langle x^{2}, y^{2}\right\rangle \cong D_{2 n}$, so there must be more than one non-involution in any generating set for $G$. This implies that we have case (B2) of Proposition 2.1, but then $m_{1}$ and $m_{2}$ must be at least 4 , and so

$$
g \geq 1+8 n(1-1 / 4-1 / 4)=1+4 n>4 n-3 .
$$

Hence we can do no better than genus $4 n-3$, and the theorem holds.

Theorems 3.1 and 3.2 together show that the symmetric genus of a finite group can be any odd positive integer.

\section{Even genera}

In this section we begin by considering another family of groups, namely 


$$
W_{n}=\left\langle x, y \mid x^{2}=y^{3 n}=1,(x y)^{3}=y^{3}\right\rangle, \text { for } n \in \mathbb{Z}^{+} .
$$

Here the relation $(x y)^{3}=y^{3}$ implies that $y^{3}$ is centralized by both $y$ and $x y$, and so is central in $W_{n}$. Thus $W_{n}$ is an extension of the central cyclic subgroup $\left\langle y^{3}\right\rangle$ by the $(2,3,3)$ triangle group $\left\langle x, y \mid x^{2}=y^{3}=(x y)^{3}=1\right\rangle \cong A_{4}$, and in particular, this shows that $\left|W_{n}\right| \leq 12 n$. On the other hand, the abelianisation $W_{n} / W_{n}^{\prime}$ is cyclic of order $3 n$ (generated by the image of $y$ ). It follows that $\left|W_{n}\right|=12 n$, with $y$ having order $3 n$ (and $W_{n}^{\prime}$ having order 4 ).

Theorem 4.1. $\sigma\left(W_{n}\right)=3 n-3$ for every odd positive integer $n$.

Proof. Let $G=W_{n}$. The generating set $\left\{x, y,(x y)^{-1}\right\}$ gives an action of $G$ with signature $(0 ;+;[2,3 n, 3 n] ;\{-\})$ on an orientable surface of genus

$$
1+6 n(1-1 / 2-1 /(3 n)-1 /(3 n))=3 n-3 .
$$

When $n=1$ this action is spherical, so we will suppose that $n>1$, and that $G$ has a faithful action on some surface $S$ of genus smaller than $3 n-3$. Again Proposition 2.1 applies, but since the abelianisation $G / G^{\prime}$ is cyclic of order $3 n$, the group $G$ has no subgroup of index 2 , and so all surface actions of $G$ preserve orientation. Hence we need only consider case (A).

If $r=3$, we may assume that $2 \leq m_{1} \leq m_{2} \leq m_{3}$. If $m_{1}=2$ then since $G / G^{\prime}$ is cyclic of odd order $3 n$, we would have $m_{3} \geq m_{2} \geq 3 n$, giving $g \geq 3 n-3$. Similarly, if $m_{1}=3$ then since $G /\left\langle y^{3}\right\rangle \cong A_{4}$ we must have $\left\{m_{2}, m_{3}\right\}=\{2 s, 3 t\}$ for some $s, t$, while also since $G / G^{\prime} \cong C_{3 n}$, both $s$ and $t$ must be divisible by $n$; hence one of $m_{2}$ and $m_{3}$ is at least $2 n$ while the other is at least $3 n$, giving $g \geq 1+6 n(1-1 / 3-1 /(2 n)-1 /(3 n))=$ $4 n-4>3 n-3$. Next, $m_{1}$ cannot be 4 or 5 , since $y^{3}$ has odd order and $G /\left\langle y^{3}\right\rangle \cong A_{4}$, which has no element of order 4 or 5 . Finally, if $m_{1} \geq 6$ then $g \geq 1+6 n(1-1 / 6-1 / 6-$ $1 / 6)=3 n+1>3 n-3$. Hence the case $r=3$ is impossible.

With $r=4$, we require two of the $m_{i}$ to be 2 (otherwise $\xi \geq 2-1 / 2-3 / 3=1 / 2$ ), but then the other two must be divisible by $3 n$ since $G$ has abelianisation of odd order $3 n$, so $g \geq 1+6 n(2-2 / 2-2 /(3 n))=6 n-3>3 n-3$, which again is impossible.

If $G$ is a finite group and $H$ is a subgroup of $G$, then clearly $\sigma(H) \leq \sigma(G)$. Moreover, if $\sigma(H)=g$ and $G$ has a faithful action on a closed orientable surface of genus $g$, then $\sigma(G)=\sigma(H)$. These observations give us two further families of groups of symmetric genus divisible by 6 .

Corollary 4.2. For every odd positive integer n, each of the following groups has symmetric genus $3 n-3$ :

(a) $\left\langle u, v \mid u^{4}=v^{3 n}=(u v)^{2}=1, u^{-1} v^{3} u=v^{-3}\right\rangle$, which is an extension of $C_{n}$ by $S_{4}$, of order $24 n$, and

(b) $\left\langle a, b, c \mid a^{2}=b^{2}=c^{2}=(a b)^{4}=(b c)^{3 n}=(a c)^{2}=\left[a,(b c)^{3}\right]=1\right\rangle$, which is an extension of $C_{n}$ by $S_{4} \times C_{2}$, of order $48 n$.

Proof. If $G$ is the group in (a), then the subgroup $H$ generated by $x=u^{2}$ and $y$ has index 2 in $G$, and as the relation $(u v)^{2}=1$ implies that $x y=u^{2} v=u v^{-1} u^{-1}$, we see that $x y$ is conjugate to $v^{-1}=y^{-1}$, and further, $(x y)^{3}=u v^{-3} u^{-1}=v^{3}=y^{3}$. An easy application of Reidemeister-Schreier theory (see [22] for example) now shows that $H$ has the same 
presentation as $W_{n}$, so $|G|=24 n$. The quotient of $G$ by the cyclic normal subgroup $\left\langle v^{3}\right\rangle$ is the $(2,3,4)$ triangle group $S_{4}$, and the generating set $\{u v, u, v\}$ gives an orientationpreserving action of $G$ with signature $(0 ;+;[2,4,3 n] ;\{-\})$ on an orientable surface of genus $1+12 n(1-1 / 2-1 / 4-1 /(3 n))=3 n-3$ as well.

Similarly, if $L$ is the group in (b), then the subgroup generated by $u=a b$ and $v=b c$ has index 2 in $L$, and has the same presentation as $G$, so $|L|=48 n$. The quotient of $L$ by the cyclic normal subgroup $\left\langle(b c)^{3}\right\rangle$ is the extended $(2,3,4)$ triangle group $S_{4} \times C_{2}$ (the full symmetry group of the regular octahedron), and the generating set $\{c, a, b\}$ gives a reflective action of $G$ with signature $(0 ;+;[-] ;\{(2,4,3 n)\})$ on an orientable surface of genus $1+24 n(1 / 2-1 / 4-1 / 8-1 /(6 n))=3 n-3$.

Our next two theorems use direct products for genera congruent to $4 \bmod 6$.

Theorem 4.3. $\sigma\left(C_{n} \times S_{4}\right)=3 n+1$ for every odd integer $n \geq 5, n \neq 9$.

Proof. Let $G=C_{n} \times S_{4}$, where $n$ is odd and $n \geq 5$. In this group of order $24 n$, let $u=$ $(1,(1,3)), v=\left(1,(1,2)\right.$, and $w=(z,(1,2)(3,4))$, where $z$ is a generator for $C_{n}$. Then $u, v$ and $w$ generate $G$, since $w^{2}$ generates $C_{n}$ while the images of $w u=(z,(1,2,3,4))$ and $v$ in $G / C_{n}$ generate $S_{4}$. Also $u, v$ and $w$ satisfy $u^{2}=v^{2}=[u, w]=[v, w]^{2}=1$, and the index 2 subgroup $C_{n} \times A_{4}$ of $G$ contains $w$ but neither $u$ nor $v$. It follows that $G$ has a reflective action with signature $(0 ;+;[-] ;\{(1),(2)\})$ on an orientable surface of genus $1+12 n(1 / 2-1 / 4)=3 n+1$; this is case (B3) of Proposition 2.1.

Now suppose $G$ has a faithful action on a surface $S$ of genus $g$ smaller than $3 n+1$. Then $g<1+|G| / 8$, so we consider the cases of Proposition 2.1 with $\xi<1 / 4$. Note that the abelianisation of $G$ is $C_{2 n}$, generated by the image of $u w$ or $v w$, and the centre of $G$ is $C_{n}$, with quotient $G / Z(G) \cong S_{4}$.

Consider first case (A). If $r=3$ and one branch point has order 2 , then by considering the projections onto $C_{n}$ and $S_{4}$ we see that the other two must have orders $3 n$ and $4 n$, giving $g \geq 1+12 n(1-1 / 2-1 /(3 n)-1 /(4 n))=6 n-6>3 n+1$. Similarly, if one branch point has order 3 , then the other two must have orders at least $2 n / 3$ and $4 n / 3$, giving $g \geq 1+12 n(1-1 / 3-3 /(2 n)-3 /(4 n))=8 n-26>3 n+1$ for $n>5$, while for the remaining case $n=5$ the other two must have orders at least $2 n$ and $4 n$, giving $g \geq 1+12 n(1-1 / 3-1 /(2 n)-1 /(4 n))=8 n-8>3 n+1$. If all branch points have order 4 or more, then $g \geq 1+12 n(1-1 / 4-1 / 4-1 / 4)=3 n+1$, but abelianisation shows this bound cannot be attained when $n>2$. On the other hand, if $r=4$, then at least two branch points must have order 2 , but then the orders of the other two are divisible by $n$, so $g \geq 1+12 n(2-1 / 2-1 / 2-1 / n-1 / n)=12 n-23>3 n+1$.

In case (B1), the group $G$ is generated by an element of order $m$ and $s$ involutions, and $\xi=s / 2-1 / m-\sum_{j=1}^{s} 1 /\left(2 q_{j}\right)<1 / 4$. Abelianisation gives $m \geq n \geq 5$, which forces $s=1$, so the signature is $(0 ;+;[m] ;\{(q)\})$. It follows that $G$ is generated by two elements $u$ and $v$ such that $u^{2}=v^{m}=[u, v]^{q}=1$, with $v$ lying in $C_{n} \times A_{4}$ and $u$ lying outside it. Projection onto $S_{4}$ shows the images of $u$ and $v$ must be a 2-cycle and a 3-cycle, with also the image of $[u, v]$ a 3 -cycle. Hence $m$ is divisible by 3 and $q=3$. But now $g \geq 1+12 n(1 / 2-1 / n-1 / 6)=4 n-11>3 n+1$ for all $n>12$, while for the remaining cases $n=5,7$ and 11 (not divisible by 3 ), abelianisation gives $m \geq 3 n$ and then $g \geq 1+12 n(1 / 2-1 /(3 n)-1 / 6)=4 n-3>3 n+1$.

In case (B2), the group $G$ is generated by three elements $u, v$ and $c$ such that $u^{m_{1}}=$ $v^{m_{2}}=c^{2}=[c, u v]^{q}=1$. The condition $\xi<1 / 4$ implies $1 / m_{1}+1 / m_{2}>3 / 4$ and so 
(without loss of generality) $m_{1}=2$, but then $G$ cannot have abelianisation of order $2 n$. In cases (B3) and (C2), we have $\xi=1 / 2-1 /(2 q)<1 / 4$, which forces $q=1$, but this is impossible (by Proposition 2.1) since $G / Z(G)$ is neither cyclic nor dihedral. Case (C3) is ruled out for the same reason.

Finally, in case (C1) the group $G$ is generated by two elements $d$ and $u$ such that $u^{m_{1}}=\left(d^{2} u\right)^{m_{2}}=1$. As in case (B2), the condition $\xi<1 / 4$ gives $1 / m_{1}+1 / m_{2}>3 / 4$, so (without loss of generality) $m_{1}=2$, and then in the abelianisation the image of $d^{2}$ must have order $n$, so the image of $d^{2} u$ has order $2 n$, giving $m_{2} \geq 2 n$. This, however, implies $g \geq 1+12 n(1-1 / 2-1 /(2 n))=6 n-5>3 n+1$, another contradiction.

It is easy to prove that the symmetric group $S_{5}$ has symmetric genus 4 ; indeed $S_{5}$ and $S_{5} \times C_{2}$ are the only finite groups of symmetric genus 4 ; see Section 6. To cover the remaining cases of genus $3 n+1$ for $n=3$ and 9 , we have the following:

Theorem 4.4. $\sigma\left(C_{3} \times C_{3} \times C_{3 n}\right)=18 n-8$ for every positive integer $n$.

Proof. This is similar to previous theorems, but does not use Proposition 2.1. First, let $u, v, w$ be respective generators of the three factors of of $G=C_{3} \times C_{3} \times C_{3 n}$, and $z=(u v w)^{-1}$. Then taking $\{u, v, w, z\}$ as generating set gives an orientation-preserving action of $G$ with signature $(0 ;+;[3,3,3 n, 3 n] ;\{-\})$ on a surface of genus $1+(27 n / 2)(2-$ $1 / 3-1 / 3-1 /(3 n)-1 /(3 n))=18 n-8$. Clearly, no orientation-preserving action can do better, since $G$ has rank 3 and adding extra generators simply increases the genus. The fact that $G$ has only one involution can be used to eliminate potentially better cases where the action does not preserve orientation. We leave the details to the reader. See also [24, 27].

We note that the same argument can be used to show that

$$
\sigma\left(C_{m} \times C_{m} \times C_{m n}\right)=1+m^{2}((m-1) n-1) \quad \text { for all odd } m \text { and all } n .
$$

When $m>3$, however, this is not very useful for filling gaps in the symmetric genus spectrum; for example, when $m=5$ it covers only genera congruent to $76 \bmod 100$.

Our final theorem in this section begins to cover the gaps for genus $g \equiv 2 \bmod 6$. For this, we take the following family of groups:

$$
T_{n}=\left\langle x, y \mid x^{2}=y^{3 n}=\left[x, y^{3}\right]=1,(x y)^{3}=(y x)^{3}\right\rangle, \text { for } n \in \mathbb{Z}^{+} .
$$

We note that the abelianisation of $T_{n}$ is $C_{2} \times C_{3 n}$, and it follows that the order of $y$ is exactly $3 n$. Also $y^{3}$ is central, and $T /\left\langle y^{3}\right\rangle \cong T_{1}$.

The group $T_{1}$ has order 48 , with derived subgroup $T_{1}{ }^{\prime}$ isomorphic to the quaternion group $Q_{8}$. To see this, observe that the subgroup of index 2 in $T_{1}$ generated by $X=x y x$ and $Y=y$ has presentation $\left\langle X, Y \mid X^{3}=Y^{3}=1, X Y X=Y X Y\right\rangle$ and is isomorphic to $\mathrm{SL}(2,3)$ via the mapping $X \mapsto\left(\begin{array}{rr}0 & 1 \\ -1 & -1\end{array}\right)$ and $Y \mapsto\left(\begin{array}{rr}-1 & 1 \\ -1 & 0\end{array}\right)$. (In fact $T_{1}$ and $\operatorname{SL}(2,3)$ are two of the three groups that have (White) genus 1 but not symmetric genus 1 , as given in [18, Section 6.4]; see our comments just before Theorem 6.2.) Here $[X, Y]$ maps to $\left(\begin{array}{rr}-1 & 1 \\ 1 & 0\end{array}\right)$, which has order 4 and together with its conjugates generates a subgroup isomorphic to $Q_{8}$.

Thus $\left|T_{n}\right|=n\left|T_{1}\right|=48 n$, and also $T_{n}{ }^{\prime}$ (of index $6 n$ in $T_{n}$ ) is isomorphic to $Q_{8}$, for all $n$. It follows that the commutator of every two elements of $T_{n}$ has order 1,2 or 4 , and 
the commutator of every generating pair for $T_{n}$ has order 4 . In particular, $[x, y]\left(=X^{-1} Y\right)$ has order 4 , and $z=[x, y]^{2}$ is a central involution. Furthermore, the subgroup $K$ generated by $y^{3}$ and $z$ is central in $T_{n}$, with quotient $\operatorname{PSL}(2,3) \cong A_{4}$. Since $A_{4}$ has trivial centre, it follows that $K=Z\left(T_{n}\right)$ and $T_{n} / Z\left(T_{n}\right) \cong A_{4}$.

Theorem 4.5. $\sigma\left(T_{n}\right)=9 n-7$ for every odd positive integer $n$.

Proof. Let $G=T_{n}$, where $n$ is odd. Since the index 2 subgroup generated by $y$ and $x y x$ contains $y$ but not $x$, the relations $y^{3 n}=x^{2}=[x, y]^{4}=1$ imply that $G$ has a reflective action with signature $(0 ;+;[3 n] ;\{(4)\})$ on an orientable surface of genus

$$
1+24 n(1 / 2-1 /(3 n)-1 / 8)=9 n-7 .
$$

Now suppose $G$ has a faithful action on a surface $S$ of genus $g<9 n-7$. Again we can use Proposition 2.1 (since $\left|T_{n}\right|=48 n>4(9 n-7)$ ).

First consider case (A). If $r=3$ and $u, v, w$ are the corresponding generators for $G$ of orders $m_{1} \leq m_{2} \leq m_{3}$ and satisfying $u v w=1$, then it is not difficult to verify (with the help of MAGMA [3] for example) that the images of $u, v, w$ in the quotient $T_{1}$ must have orders $2,3,12$, or $2,6,12$, or $3,12,12$, or $4,12,12$, or $6,12,12$. If $m_{1}=2$, then abelianisation shows that $m_{2} \geq 3 n$ and $m_{3} \geq 12 n$. Thus $g \geq 1+24 n(1-1 / 2-1 /(3 n)-$ $1 /(12 n))=12 n-10>9 n-7$. Similarly, if $m_{1}=3,4$ or 6 then $m_{3} \geq m_{2} \geq 12 n$, giving $g \geq 1+24 n(1-1 / 3-1 /(12 n)-1 /(12 n))=16 n-3>9 n-7$, and if $m_{1} \geq 7$ then $g \geq 1+24 n(1-1 / 7-1 / 7-1 / 7)=(96 / 7) n+1>9 n-7$. Similarly, if $r=4$ then at least two branch points must have order 2 , but then the orders of the other two branch points must be divisible by $3 n$ (by abelianisation), so $g \geq 1+24 n(2-1 / 2-1 / 2-1 /(3 n)-1 /(3 n))=$ $24 n-15>9 n-7$. Hence no such action gives genus smaller than $9 n-7$.

In case (B1), the group $G$ is generated by an element of order $m$ and $s$ involutions, and $\xi=s / 2-1 / m-\sum_{j=1}^{s} 1 /\left(2 q_{j}\right)<1 / 2$. Abelianisation gives $m \geq 3 n$, which then forces $s=1$ (since we may suppose $q_{j} \geq 2$ for all $j$ when $s>1$ ). Thus $G$ is generated by two elements $u$ and $v$ such that $u^{2}=v^{m}=[u, v]^{q}=1$, and necessarily $m \geq 3 n$ and $q \geq 4$ (by our comments above about $T_{n}{ }^{\prime}$ ), so we can do no better than our specified action of genus $9 n-7$.

In case (B2), the group $G$ has generators $u, v$ and $c$ such that $u^{m_{1}}=v^{m_{2}}=c^{2}=$ $[c, u v]^{q}=1$, and $g=1+24 n\left(3 / 2-1 / m_{1}-1 / m_{2}-1 / q\right) \geq 1+24 n\left(1-1 / m_{1}-1 / m_{2}\right)$. In order to make this less than $9 n-7$ we require $1 / m_{1}+1 / m_{2}>15 / 24$, so without loss of generality $m_{1}$ is 2 or 3 . But if $m_{1}=2$ then abelianisation gives $m_{2} \geq 3 n$, so that $g \geq 1+24 n(1-1 / 2-1 /(3 n))=12 n-7>9 n-7$. Similarly, if $m_{1}=3$ then $m_{2} \geq n$, and so for $n>1$ we have $g \geq 1+24 n(1-1 / 3-1 / n)=16 n-23>9 n-7$, while for $n=1$ we have $m_{2} \geq 2$ (since the orders of interior branch points are always at least 2 ) and then $g \geq 1+24(1-1 / 3-1 / 2)=5>9 n-7$.

In case (B3), the group $G$ is generated by three elements $u, v$ and $w$ such that $u^{2}=$ $v^{2}=[u, w]=[v, w]^{q}=1$, and $g=1+24 n(1 / 2-1 / 2 q)$. To make this less than $9 n-7$ we require $q<4$, so $q=1$ or 2 . Now $q \neq 1$ since $G / Z(G) \cong A_{4}$ is neither cyclic nor dihedral; hence $q=2$. But then $[v, w]$ is the unique involution $z$ in $G^{\prime} \cong Q_{8}$, so the quotient $\bar{G}=G /\langle z\rangle$ is generated by $\bar{u}$ and $\bar{v}$ (of order 1 or 2 ) and the central element $\bar{w}$, which is impossible since $G / Z(G) \cong A_{4}$ is not generated by involutions.

In case (C1), the group $G$ is generated by $d$ and $u$ such that $u^{m_{1}}=\left(d^{2} u\right)^{m_{2}}=1$, and $g=1+24 n\left(1-1 / m_{1}-1 / m_{2}\right)$. For this to be less than $9 n-7$ we require $1 / m_{1}+$ $1 / m_{2}>15 / 24$, so without loss of generality $m_{1}$ is 2 or 3 . But if $m_{1}=2$ then in the 
abelianisation $C_{6 n}$ the image of $d^{2}$ must have (odd) order $3 n$, so $m_{2} \geq 3 n$, which gives $g \geq 1+24 n(1-1 / 2-1 /(3 n))=12 n-7>9 n-7$. Similarly, if $m_{1}=3$ then in the (cyclic) abelianisation $C_{6 n}$ the order of the image of $d^{2}$ must be $n$ or $3 n$, and then so must the order of the image of $d^{2} u$ (whether 3 divides $n$ or not), and thus $m_{2} \geq n$. This gives $g \geq 1+24 n(1-1 / 3-1 / n)=16 n-23>9 n-7$ for $n>1$, while for $n=1$ we have $m_{2} \geq 2$ (since the orders of interior branch points are always at least 2 ) and then $g \geq 1+24(1-1 / 3-1 / 2)=5>9 n-7$.

In case (C2), the group $G$ has two generators $d$ and $c$ such that $c^{2}=\left[d^{2}, c\right]^{q}=1$, and just as in the case (B3), we find $q=2$. But then $\left[d^{2}, c\right]$ is the unique involution $z$ in $G^{\prime} \cong Q_{8}$, and in the quotient $\bar{G}=G /\langle z\rangle$, the image of $d^{2}$ is central, so $G / Z(G) \cong A_{4}$ is generated by the involutory images of $c$ and $d$, which is again impossible.

Finally, the case (C3) is ruled out also by the fact that $G / Z(G) \cong A_{4}$ is neither cyclic nor dihedral (or since $G$ has no abelian subgroup of index 2 ).

The first theorems in this section cover all genera $g \equiv 0$ or $4 \bmod 6$, and the last one covers all $g \equiv 2 \bmod 18$. All that remains are genera $g \equiv 8$ or $14 \bmod 18$, and thus we have proved the first part of Theorem 1.2.

\section{$5 \quad$ Filling some gaps}

Let $A$ be any finite abelian group that admits an automorphism $\theta$ of order 3 , with the property that for some $x \in A$, the element $x$ and its images $x^{\theta}$ and $x^{\theta^{2}}$ generate $A$, and satisfy $x x^{\theta} x^{\theta^{2}}=1$. Now form the semi-direct product $A \rtimes_{\theta} C_{6}$, in which conjugation by a generator $y$ of $C_{6}$ induces the automorphism $\theta$ on $A$. This group has order $6|A|$, and the element $y^{3}$ is a central involution (so $A \rtimes_{\theta} C_{6} \cong\left(A \rtimes_{\theta} C_{3}\right) \times C_{2}$ ). Also $x y^{-1}$ has order 6 , since $\left(x y^{-1}\right)^{3}=x\left(y^{-1} x y\right)\left(y^{-2} x y^{2}\right) y^{-3}=x x^{\theta} x^{\theta^{2}} y^{3}=y^{3}$, which has order 2 ; similarly $x y^{-2}$ has order 3 . Hence the elements $x_{1}=x y^{-2}, x_{2}=y$ and $x_{3}=y x^{-1}=\left(x y^{-1}\right)^{-1}$ satisfy the relations $x_{1}{ }^{3}=x_{2}{ }^{6}=x_{3}{ }^{6}=x_{1} x_{2} x_{3}=1$, and give rise to a faithful action of $A \rtimes_{\theta} C_{2}$ on an orientable surface of genus

$$
1+3|A|(1-1 / 3-1 / 6-1 / 6)=|A|+1 .
$$

There are several ways to obtain such an abelian group $A$ and automorphism $\theta$. For example, if $p$ is any prime congruent to $1 \bmod 3$, and $e$ is any positive integer, then the group of units mod $p^{e}$ has order $\phi\left(p^{e}\right)=p^{e-1}(p-1) \equiv 0 \bmod 3$, so there exists a non-trivial cube root of $1 \bmod p^{e}$, with $1+\lambda+\lambda^{2} \equiv 0 \bmod p^{e}$, and then we can take $A=C_{p^{e}}=\left\langle x \mid x^{p^{e}}=1\right\rangle$ and $\theta$ the automorphism taking $x \mapsto x^{\lambda}$. Alternatively, if $m$ is any positive integer, we can take $A=C_{m} \times C_{m}=\left\langle x, z \mid x^{m}=z^{m}=[x, z]=1\right\rangle$ and let $\theta$ be the automorphism taking $x \mapsto z \mapsto x^{-1} z^{-1} \mapsto x$. More generally, we have the following:

Theorem 5.1. Let $A$ be a non-trivial abelian group of the form $C_{s} \times C_{t} \times C_{t}$, where $s$ and $t$ are odd, $\operatorname{gcd}(s, t)=1$, and every prime divisor of $s$ is congruent to $1 \bmod 3$. Then $A$ has an automorphism $\theta$ of order 3 with the property that $\sigma\left(A \rtimes_{\theta} C_{6}\right)=|A|+1$.

Proof. For each maximal prime-power divisor $q_{i}=p_{i}^{e_{i}}$ of $s$, there exists a non-trivial cube root $\lambda_{i}$ of $1 \bmod q_{i}$, with $1+\lambda_{i}+\lambda_{i}^{2} \equiv 0 \bmod q_{i}$, and then by the Chinese remainder theorem, there exists an integer $\lambda$ such that $\lambda \cong \lambda_{i} \bmod q_{i}$ for all $i$, from which it follows 
that also $1+\lambda+\lambda^{2} \equiv 0 \bmod s$. Now if $\{u, v, w\}$ is the standard basis for $A$, we may define the automorphism $\theta$ by setting

$$
u^{\theta}=u^{\lambda}, \quad v^{\theta}=w, \quad w^{\theta}=v^{-1} w^{-1} .
$$

It is easy to see that $\theta$ has order 3 , and also that if $x=u v$, we have

$$
x x^{\theta} x^{\theta^{2}}=(u v)\left(u^{\lambda} w\right)\left(u^{\lambda^{2}} v^{-1} w^{-1}\right)=u^{1+\lambda+\lambda^{2}} v w v^{-1} w^{-1}=1 .
$$

Moreover, since $\operatorname{gcd}(s, t)=1$, the element $x=u v$ generates $C_{s} \times C_{t}$, and so $x$ and $x^{\theta}=u^{\lambda} w$ generate $A$. Hence by the observations made earlier, the group $G=A \rtimes_{\theta} C_{6}$ has a faithful action on a surface of genus $|A|+1$.

Now suppose $G$ has a faithful action on a surface $S$ of genus $g<|A|+1$. Again Proposition 2.1 applies.

Here the fact that $G \cong\left(A \rtimes_{\theta} C_{3}\right) \times C_{2}$ has a unique involution $z$ (with non-cyclic quotient $G /\langle z\rangle \cong A \rtimes_{\theta} C_{3}$ ) implies that any generating set for $G$ must contain at least two elements of order greater than 2. In particular, this rules out cases (B1), (B3) and (C2).

Next consider case (A). If $r=3$, let $u, v, w$ be the corresponding generators for $G$ of orders $m_{1}, m_{2}, m_{3}$ and satisfying $u v w=1$. Then since any two of $u, v, w$ generate $G$, their orders must all be at least 3 , and since $G / A \cong C_{6}$, at least two must be 6 or more, so $g \geq 1+3|A|(1-1 / 3-1 / 6-1 / 6)=|A|+1$. Similarly if $r=4$ then at least two of the $m_{i}$ are at least 3 , so $g \geq 1+3|A|(2-1 / 2-1 / 2-1 / 3-1 / 3) \geq|A|+1$.

In case (B2), where $G$ has generators $u, v$ and $c$ satisfying $u^{m_{1}}=v^{m_{2}}=c^{2}=$ $[c, u v]^{q}=1$, we have $m_{1}, m_{2} \geq 3$, so $g \geq 1+3|A|(1-1 / 3-1 / 3)=|A|+1$. Similarly, in case (C1), the group $G$ is generated by $d, u$ and $v$ such that $u^{m_{1}}=v^{m_{2}}=d^{2} u v=1$. Here we have $G=\langle d, u\rangle=\left\langle d, d^{2} u\right\rangle$, so both $u$ and $v$ have order greater than 2 , which again gives $g \geq 1+3|A|(1-1 / 3-1 / 3)=|A|+1$.

Finally, case (C3) is impossible since $G$ has no abelian subgroup of index 2 .

Note that a similar construction was used in [12, §3.4.1] for groups acting on nonorientable surfaces.

Corollary 5.2. Let $g$ be an even positive integer, such that $g-1=p_{1}^{e_{1}} p_{2}^{e_{2}} \ldots p_{m}{ }^{e_{m}}$ where the $p_{i}$ are distinct primes greater than 3 (and the $e_{i}$ are positive integers). If $p^{e_{i}} \not \equiv 5$ mod 6 for $1 \leq i \leq m$, then there exists a finite group of symmetric genus $g$.

Proof. By the given condition, we can write $g-1$ as $s t^{2}$, where all prime divisors of $s$ are congruent to $1 \bmod 6$, and then $\sigma\left(C_{s} \times C_{t} \times C_{t}\right)=g$ by the above theorem.

Note that this completes the proof of Theorem 1.2, since if $g \equiv 8$ or $14 \bmod 18$ then every prime divisor of $g-1$ must be congruent to $\pm 1 \bmod 6$.

Corollary 5.3. Let $A$ be as in Theorem 5.1. Then there exists a semi-direct product $A$ : $\left(C_{6} \times C_{2}\right)$ having symmetric genus $|A|+1$.

Proof. Instead of $\theta$, take the automorphism $\psi$ of $A=C_{s} \times C_{t} \times C_{t}$ given by

$$
u^{\psi}=u^{\xi}, \quad v^{\psi}=w, \quad w^{\theta}=v^{-1} w
$$

where $\xi$ is an integer satisfying $\xi^{2}-\xi+1 \equiv 0 \bmod s$ (so that $\xi^{3} \equiv-1 \bmod s$ ), whose existence is guaranteed by the assumption that $p^{e_{i}}-1 \equiv 0 \bmod 6$ for every maximal primepower divisor $p_{i}^{e_{i}}$ of $s$. Now form the semi-direct product $G=A:\left(C_{6} \times C_{2}\right)$ by letting a 
generator $y$ of the factor $C_{6}$ induce the automorphism $\psi$ on $A$ and letting a generator $z$ of the factor $C_{2}$ centralize $A$. In this group $G$, take the elements

$$
x_{1}=u v y^{3} z, \quad x_{2}=y, \quad \text { and } \quad x_{3}=\left(u v y^{4} z\right)^{-1} .
$$

Since $z$ centralizes $A$ and conjugation by $y^{3}$ inverts every element of $A$, we have $x_{1}^{2}=$ $u v u^{-1} v^{-1}=1$, and similarly $\left(u v y^{4} z\right)^{3}=u^{1+\xi^{2}+\xi^{4}}\left(v\left(v^{-1} w\right) w^{-1}\right) y^{6} z^{3}=u^{1-\xi+\xi^{2}} z=$ $z$, so $x_{1}, x_{2}$ and $x_{3}$ have orders 2, 6 and 6 respectively. Moreover, the subgroup generated by $x_{1}, x_{2}$ and $x_{3}$ contains $y=x_{2}$ and $z=x_{3}{ }^{3}$ and so contains also $u v=x_{1} z y^{3}$ and $u^{\xi} w=$ $(u v)^{y}$, so contains all of $G$. Thus $G$ has a faithful action on an orientable surface of genus $1+6|A|(1-1 / 2-1 / 6-1 / 6)=|A|+1$. Finally, since $1+\xi^{2}+\left(\xi^{2}\right)^{2} \equiv 1-\xi+\xi^{2} \equiv 0$ mod $s$, the automorphism $\psi^{2}$ is the same as $\theta$ or $\theta^{-1}$, so the index 2 subgroup of $G$ generated by $A$ and $y^{2} z$ or $y^{4} z$ is isomorphic to the group $A \rtimes_{\theta} C_{6}$ considered in Theorem 5.1, and it follows that $\sigma(G)=\sigma\left(A \rtimes_{\theta} C_{6}\right)=|A|+1$.

\section{Remaining gaps in the spectrum}

Our constructions so far cover well over eight ninths of all positive integers as possibilities for $\sigma(G)$ for some finite group $G$. The smallest genera not covered by Theorem 1.2 are $g=86=5 \times 17+1, g=116=5 \times 23+1$, and $g=188=11 \times 17+1$. There are, however, no gaps in the spectrum at these three values of $g$, for :

(a) the group $\left\langle x, y \mid x^{8}=y^{51}=1, x^{-1} y x=y^{25}\right\rangle \cong C_{51} \rtimes_{25} C_{8}$ of order 408 (and with centre of order 3 ) has symmetric genus 86 , realised by an action with signature $(0 ;+;[2,24,24] ;\{-\})$;

(b) the group $\left\langle u, v, t \mid u^{25}=v^{5}=[u, v]=t^{4}=1, t^{-1} u t=u^{19} v^{4}, t^{-1} v t=u^{15} v^{2}\right\rangle$, which is a semi-direct product $\left(C_{25} \times C_{5}\right): C_{4}$ of order 125 , has symmetric genus 116 , realised by an action with signature $(0 ;+;[4,4,25] ;\{-\})$; and

(c) the group $\left\langle x, y \mid x^{10}=y^{99}=1, x^{-1} y x=y^{17}\right\rangle \cong C_{99} \rtimes_{17} C_{10}$ of order 990 (and with trivial centre) has symmetric genus 188 , realised by an action with signature $(0 ;+;[2,10,45] ;\{-\})$.

These examples (which were found with the help of MAGMA [3]) are members of further infinite families that might be helpful in closing the gaps, but the orders of groups in such infinite families do not behave as well as those considered in this and the previous two sections.

It is illuminating to consider the groups of small symmetric genus. We have used MAGMA [3] to determine all finite groups of symmetric genus 2 to 32 inclusive, and May and Zimmerman [30] have done this independently for genus 2 to 8 . Table 1 presents a summary of the groups of symmetric genus 2,8 , and 14 , which we felt could be helpful in finding further families of groups of symmetric genus congruent to $2 \bmod 6$.

The family of metabelian groups is helpful in dealing with some genera. We have found (again with the help of MAGMA [3]) that there are no metacyclic groups of symmetric genus $2,3,4,5,6,10,13,24,26,30$ or 37 . On the other hand, although there are no metabelian groups of symmetric genus 2,3 or 4 , there do exist abelian or metabelian groups of symmetric genus $g$ for all $g$ between 5 and 38. This provides evidence (however flimsy that it might be) in support of the following stronger version of Conjecture 1.3:

Conjecture 6.1. For every integer $g \geq 5$, there is a finite abelian or metabelian group $G$ with symmetric genus $\sigma(G)=g$. 


\begin{tabular}{|c|c|l|l|}
\hline$\sigma$ & $|G|$ & Description of $G$ & Signature $(\mathrm{s})$ \\
\hline 2 & 24 & $\mathrm{SL}(2,3)$ & $(0 ;+;[3,3,4] ;\{-\})$ \\
& 48 & $\mathrm{GL}(2,3)$ & $(0 ;+;[2,3,8] ;\{-\}),(0 ;+;[-] ;\{(3,3,4)\})$ \\
& 48 & $\mathrm{SL}(2,3): C_{2}$ & $(0 ;+;[3] ;\{(4)\})$ \\
& 96 & $\mathrm{GL}(2,3): C_{2}$ & $(0 ;+;[-] ;\{(2,3,8)\})$ \\
\hline 8 & 42 & $\left(C_{7} \rtimes_{2} C_{3}\right) \times C_{2}$ & $(0 ;+;[3,6,6] ;\{-\}),(1 ;-;[3,3] ;\{-\}),(0 ;+;[3,3] ;\{(1)\})$ \\
& 48 & $\mathrm{SL}(2,3): C_{2}$ & $(0 ;+;[3,4,8] ;\{-\})$ \\
& 84 & $C_{7}:\left(C_{6} \times C_{2}\right)$ & $(0 ;+;[2,6,6] ;-\}),(0 ;+;[3] ;\{(2,2)\}),(0 ;+;[2,3] ;\{(1)\})$ \\
& 96 & $\mathrm{GL}(2,3): C_{2}$ & $(0 ;+;[-] ;\{(3,4,8)\})$ \\
& 336 & $\operatorname{PSL}(2,7) \times C_{2}$ & $(0 ;+;[-] ;\{(3,3,4)\}),(0 ;+;[3] ;\{(4)\})$ \\
& 672 & $\operatorname{PGL}(2,7) \times C_{2}$ & $(0 ;+;[-] ;\{(2,3,8)\})$ \\
\hline 14 & 78 & $\left(C_{13} \rtimes_{3} C_{3}\right) \times C_{2}$ & $(0 ;+;[3,6,6] ;\{-\}),(1 ;-;[3,3] ;\{-\}),(0 ;+;[3,3] ;\{(1)\})$ \\
& 120 & $\operatorname{SL}(2,5)$ & $(0 ;+;[3,4,5] ;\{-\})$ \\
& 156 & $C_{13}:\left(C_{6} \times C_{2}\right)$ & $(0 ;+;[2,6,6] ;\{-\}),(0 ;+;[3] ;\{(2,2)\}),(0 ;+;[2,3] ;\{(1)\})$ \\
& 156 & $C_{13} \rtimes_{2} C_{12}$ & $(1 ;-;[2,3] ;\{-\})$ \\
& 240 & $\operatorname{SL}(2,5): C_{2}$ & $(0 ;+;[-] ;\{(3,4,5)\})$ \\
1092 & $\operatorname{PSL}(2,13)$ & $(0 ;+;[2,3,7] ;\{-\})$ \\
& 2184 & $\operatorname{PGL}(2,13)$ & $(0 ;+;[-] ;\{(2,3,7)\})$ \\
& 2184 & $\operatorname{PSL}(2,13) \times C_{2}$ & $(0 ;+;[-] ;\{(2,3,7)\})$ \\
\hline
\end{tabular}

Table 1: Groups of symmetric genus $\sigma(G)=2,8$ or 14

Aside from the symmetric genus, the strong symmetric genus and the symmetric crosscap number, there is another parameter known simply as the genus (or sometimes the White genus). The (White) genus $\gamma(G)$ of a finite group $G$ is the smallest non-negative integer $g$ such that some Cayley graph for the group $G$ has a 2-cell embedding in an orientable surface of genus $g$; see [33].

In general, $\gamma(G) \leq \sigma(G)$, but computation of $\gamma$ is far more difficult than $\sigma(G)$, except for very small $G$, those with $\gamma(G)=0$ or 1 , and those $G$ attaining the lower bound $\sigma(G) \geq$ $1+|G| / 168$ when $\gamma(G)>1$; see [32]. The reason for this is that Cayley graphs have many possible embeddings into surfaces, very few of which allow the vertex-transitive action of the group on the Cayley graph to induce an action of the same group on the surface. In particular, it is difficult to say much at all about the spectrum of values of the (White) genus function $\gamma$.

Here we make just one observation:

Theorem 6.2. If $g=s t^{2}+1$ for integers $s$ and $t$ with $t \geq 2$, then there exists a group of (White) genus $g$.

Proof. Let $G$ be the abelian group $C_{2 s t} \times C_{2 t} \times C_{2}$. Take two copies of the standard embedding of a 4-valent Cayley graph for $C_{2 s t} \times C_{2 t}=\left\langle x, y \mid x^{2 s t}=y^{2 t}=[x, y]=1\right\rangle$ in the torus (where edges correspond to multiplication by $x^{ \pm 1}$ or $y^{ \pm 1}$, and every face is a quadrilateral corresponding to a commutator $\left[x^{ \pm 1}, y^{ \pm 1}\right]$ ). The quadrilaterals with opposite corners $\left(x^{2 i}, y^{2 j}\right)$ and $\left(x^{2 i+1}, y^{2 j+1}\right)$ form a 2-factor in the Cayley graph, and these can be used to tube together the two copies, giving an all-quadrilateral embedding of a Cayley graph for $G$. (This is a simple example of the White-Pisanski construction; see [18] or [31].) The genus of the resulting surface is $1+(2 t)(2 s t)(1-5 / 2+5 / 4)=1+s t^{2}$. Furthermore, since the only way to have any triangular faces is to allow generators of order 3 , which would increase the valence to 7 or more, this all-quadrilateral embedding has the 
least possible genus among all embeddings of Cayley graphs for $G$. Thus $\gamma(G)=1+s t^{2}$.

Corollary 6.3. There exists a group of (White) genus $g$ whenever $g \equiv 1$ mod $t^{2}$ for some non-zero integer $t$. In particular, this occurs whenever $g \equiv 1 \bmod 4$ or 9 .

The results presented in this paper are also related to the study of regular maps on surfaces. Roughly speaking, a regular map is a 2-cell embedding of a graph or multigraph into a closed surface with a large group of symmetries (automorphisms of the graph or multigraph that preserve the faces), so that there exists automorphisms inducing a single-step local rotation about any given vertex or any given face. As such, regular maps generalise the Platonic solids (which may be viewed as regular maps on the sphere) to surfaces of higher genera.

On orientable surfaces, a regular map is called reflexible if it admits an automorphism fixing an edge (and so reversing orientation), and chiral otherwise. Some very recent research has considered the genus spectrum of these and other classes of regular maps. In [2], for example, some ground-breaking work by Breda, Nedela, and Širáň [2] showed that there are no regular maps at all on non-orientable surfaces of Euler characteristic $-p$ where $p>13$ is prime and $p \equiv 1 \bmod 12$. We took a different approach in [13], to obtain a shorter proof of the latter, and also to prove that:

(a) there is no chiral regular map on an orientable surface of genus $p+1$, where $p$ is prime and $p \not \equiv 1 \bmod 6,8,10$, and

(b) there is no reflexible regular map with simple underlying graph (that is, with no loops or multiple edges) on an orientable surface of genus $p+1$, where $p>13$ is a prime congruent to $1 \bmod 6$.

The precise genus spectra of each of these two classes of maps are still unknown.

\section{References}

[1] R. Accola, On the number of automorphisms of a closed Riemann surface, Trans. Amer. Math. Soc. 131 (1968), 398-408.

[2] A.B. D’Azevedo, R. Nedela and J. Širáň, Classification of regular maps of negative prime Euler characteristic, Trans. Amer. Math. Soc. 357 (2005), 4175-4190.

[3] W. Bosma, J. Cannon and C. Playoust, The Magma Algebra System I: The User Language, J. Symbolic Computation 24 (1997), 235-265.

[4] H. R. Brahana, Regular maps and their groups, Amer. J. Math. 49 (1927), 268-284.

[5] W. Burnside, Theory of Groups of Finite Order, Cambridge University Press, 1911

[6] M. D. E. Conder, Generators of the alternating and symmetric groups, J. London Math. Soc. (2) 22 (1980), 75-86.

[7] M. D. E. Conder, Some results on quotients of triangle groups. Bull. Austral. Math. Soc. 30 (1984), 73-90.

[8] M. D. E. Conder, The symmetric genus of alternating and symmetric groups, J. Combin. Theory Series B 39 (1985), 179-186.

[9] M. D. E. Conder, Hurwitz groups: a brief survey, Bull. Amer. Math. Soc. 23 (1990), 359-370.

[10] M. D. E. Conder and P. Dobcsányi, Applications and adaptations of the low index subgroups procedure, Math. Comp. 74 (2005), 485-497. 
[11] M. D. E. Conder, R. Jajcay and T. W. Tucker, Regular Cayley maps for abelian groups, J. Algebraic Combinatorics 25 (2007), 259-283.

[12] M. Conder, C. Maclachlan, S.T. Vasiljevic and S. Wilson, Bounds for the number of automorphisms of a compact non-orientable surface, J. London Math. Soc. (2) 68 (2003), 65-82.

[13] M. Conder, J. Širáň and T. W. Tucker, The genera, reflexibility and simplicity of regular maps. J. Eur. Math. Soc. 12 (2010), 343-364.

[14] M. D. E. Conder, R. A. Wilson and A. J. Woldar, The symmetric genus of sporadic groups. Proc. Amer. Math. Soc. 116 (1992), 653-663.

[15] H. S. M. Coxeter and W. O. J. Moser, Generators and Relations for Discrete Groups, 4th ed., Springer Berlin (1980).

[16] J. J. Etayo Gordejuela and E. Martinez, The symmetric cross-cap number of the groups $C_{m} \times$ $D_{n}$, Proc. Roy. Soc. Edin. 138 (2008), 1197-1213.

[17] H. H. Glover and D. Sjerve, The genus of $\mathrm{PSL}_{2}$ (q), J. Reine Angew. Math. 380 (1987), 59-86.

[18] J. L. Gross and T.W. Tucker, Topological Graph Theory, Dover, 2001 (original Wiley, 1987).

[19] W. J. Harvey, Cyclic groups of automorphisms of a compact Riemann surface, Quarterly J. Math. Oxford 17 (1964), 86-97.

[20] A. Hurwitz, Über algebraische Gebilde mit eindeutigen Transformationen in sich, Math. Ann. 41 (1893), 403-442.

[21] M. A. Jackson, The strong symmetric genus of the finite Coxeter groups, J. Group Theory 10 (2007), 841-847.

[22] D. L. Johnson, Presentations of Groups, 2nd ed., Cambridge Univ. Press (1997).

[23] G. A. Jones and D. Singerman, Complex functions. An algebraic and geometric viewpoint, Cambridge University Press, 1987.

[24] C. Maclachlan, Abelian groups of automorphisms of compact Riemann surfaces, Proc. London Math. Soc. 15 (1965), 699-712.

[25] C. Maclachlan, A bound for the number of automorphisms of compact Riemann surface, $J$. London Math. Soc. 44 (1969), 265-272.

[26] H. Maschke, The representation of finite groups, especially of the rotation groups of the regular bodies of three- and four-dimensional space, by Cayley's color diagrams, Amer. J. Math. 18 (1896), 156-194.

[27] C. L. May and J. Zimmerman, The symmetric genus of finite abelian groups, Illinois J. Math. 37 (1993), 400-423.

[28] C. L. May and J. Zimmerman, There is a group of every strong symmetric genus, Bull. London Math. Soc. 35 (2003), 433-439.

[29] C. L. May and J. Zimmerman, The symmetric genus of groups of odd order, Houston J. Math. 34 (2008), 319-338.

[30] C. L. May and J. Zimmerman, The groups of symmetric genus $\sigma \leq 8$, Comm. Algebra 36 (2008), 4078-4095.

[31] T. Pisanski and A. T. White, Non-orientable embeddings of groups, Europ. J. Combin. 9 (1988), 445-461.

[32] T. W. Tucker, Finite groups acting on surfaces and the genus of a group, J. Combin. Theory (B) 34 (1983), 82-98.

[33] A. T. White, Graphs, Groups and Surfaces, (Rev. Ed.), North-Holland, 1984 
[34] R. A. Wilson, The Monster is a Hurwitz group, J. Group Theory 4 (2001), 367-374.

[35] A. Wiman, Über die hyperelliptischen Curven und diejenigen vom Geschlechte $p=3$, welche eindeutigen Transformationen in sich zulassen, Bihang Kongl. Svenska Vetenskaps-Akademiens Handlingar (Stockholm, 1895-96). 